DISEÑO CURRICULAR DE UN NUEVO POSGRADO DE NEGOCIOS CON UN ENFOQUE DE INNOVACIÓN, COMPETITIVIDAD, RESPONSABILIDAD SOCIAL Y ÉTICA EMPRESARIAL

\title{
DISEÑO CURRICULAR DE UN NUEVO POSGRADO DE NEGOCIOS CON UN ENFOQUE DE INNOVACIÓN, COMPETITIVIDAD, RESPONSABILIDAD SOCIAL Y ÉTICA EMPRESARIAL
}

\section{CURRICULAR DESIGN OF A NEW BUSINESS POSTGRADUATE WITH A FOCUS ON INNOVATION, COMPETITIVENESS, SOCIAL RESPONSIBILITY AND BUSINESS ETHICS}

\author{
Esther Castañón Nieto*, María del Consuelo Ávila Ortega**,

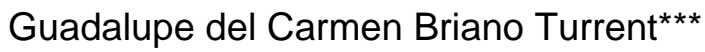

\footnotetext{
* Maestra en Administración. Facultad de Contaduría y Administración UASLP.

** Doctora. Facultad de Contaduría y Administración UASLP. consuelo.avila@uaslp.mx

*** Doctora. Facultad de Contaduría y Administración UASLP. guadalupe.briano@uaslp.mx
}

Dirección para recibir correspondencia: ecasta@uaslp.mx 
DISEÑO CURRICULAR DE UN NUEVO POSGRADO DE NEGOCIOS CON UN ENFOQUE DE INNOVACIÓN, COMPETITIVIDAD, RESPONSABILIDAD SOCIAL Y ÉTICA EMPRESARIAL

\section{RESUMEN}

OBJETIVO: Desarrollar un nuevo programa de Maestría que cumpla con los requerimientos de las instancias certificadoras y acreditadoras, así como con los programas institucionales y con las necesidades del entorno.

MATERIAL Y MÉTODO: Análisis de las tendencias a nivel nacional, regional y local de la oferta de los programas de negocios, de la demanda de los mismos, además consultas a expertos, empleadores y profesores con una metodología cualitativa para que externaran su opinión respecto a los cursos y seminarios que las fuerzas de cambio han hecho necesarios.

RESULTADOS: Un programa nuevo de Maestría que busca formar profesionales con los conocimientos y habilidades para transformar la forma actual en que trabajan las empresas con un enfoque de competitividad, ética y responsabilidad social.

CONCLUSIONES: Una vez realizados todos los estudios de oferta y demanda, así como las consultas necesarias, la División de Estudios de Posgrado de la FCA, desarrolló un nuevo programa de Maestría en Dirección Empresarial, particularmente como un programa de posgrado profesionalizante, aprovechando esta oportunidad en el mercado y atendiendo los lineamientos del modelo CONACYT de referencia.

PALABRAS CLAVE: Diseño curricular. Nuevos programas educativos de posgrado en negocios. 
DISEÑO CURRICULAR DE UN NUEVO POSGRADO DE NEGOCIOS CON UN ENFOQUE DE INNOVACIÓN, COMPETITIVIDAD, RESPONSABILIDAD SOCIAL Y ÉTICA EMPRESARIAL

\section{ABSTRACT}

OBJECTIVE: Develop a new Master's program that fulfills the requirements of the certifying and accrediting bodies, as well as those of the institutional programs and the needs of the environment.

MATERIAL AND METHOD: Analysis of national, regional and local trends regarding the supply of business programs, their demand, as well as consultations with experts, employers and teachers with a qualitative methodology to let them express their opinion about the courses and seminars that the forces of change have made necessary.

RESULTS: A new Master's program that seeks to train professionals with the knowledge and skills to transform the current way in which companies work with a focus on competitiveness, ethics and social responsibility.

CONCLUSIONS: Once all the supply and demand studies were accomplished, as well as the necessary consultations, the Postgraduate Studies Division of the FCA developed a new Master's program in Business Management Direction, particularly as a professional postgraduate program, taking advantage of this opportunity in the market and following the guidelines of the reference CONACYT model.

KEYWORDS: Curricular design. New educational postgraduate programs in business. 
DISEÑO CURRICULAR DE UN NUEVO POSGRADO DE NEGOCIOS CON UN ENFOQUE DE INNOVACIÓN, COMPETITIVIDAD, RESPONSABILIDAD SOCIAL Y ÉTICA EMPRESARIAL

\section{INTRODUCCIÓN}

La globalización y la internacionalización de los mercados han definido un entorno complejo y dinámico, lo cual demanda nuevas reglas y modelos de negocios para las organizaciones, ello incide en el perfil y la formación de los profesionistas que están al frente de las empresas, lo que viene a representar un reto importante para las instituciones de educación superior, particularmente de aquéllas que ofertan programas de posgrado en al área de negocios. Las organizaciones actuales han adoptado como pilares estratégicos a la innovación, la adopción de modelos sofisticados de negocio y el uso intensivo de tecnologías que les permitan ser más eficientes y flexibles ante un mercado global más exigente. Ello demanda que los profesionistas al mando de las empresas cuenten con las competencias y habilidades para hacer frente a los cambios incesantes, ritmos acelerados y problemáticas del entorno global, sin perder de vista la calidad, sustentabilidad, la responsabilidad social y la conducción ética de los negocios.

\section{Planteamiento del problema}

En la actualidad, las Instituciones de Educación Superior se enfrentan a una cantidad de exigencias por parte de los empleadores, las organizaciones evaluadoras y acreditadoras, los nuevos modelos y conocimientos en las diferentes profesiones y hay actores como los egresados de los programas, los expertos en el desempeño del campo profesional, los académicos, las organizaciones que contratan a los egresados y las demandas de quienes desean ingresar a un programa educativo.

Sin embargo, el planteamiento de nuevos programas educativos no siempre contempla el considerar las opiniones de todos los actores involucrados, más aún nuevos elementos como la vinculación, la movilidad nacional e internacional, la pertinencia, la cobertura, la innovación, formación holística, sustentable y ética hace aún más complejo el diseño de estos programas, no perdiendo de vista los requisitos que le permitan acreditar y posicionarse como un programa académico y una institución de calidad.

A la anterior problemática, no pueden ser ajenos los programas de posgrado de negocios, así como tampoco el entorno en que se encuentra inmersa la UASLP que debe atender los retos de las empresas que contratan a los egresados y exigen una mayor calidad, ya que en la localidad se encuentran 11 parques industriales y alrededor de 450 empresas de carácter mundial. 
DISEÑO CURRICULAR DE UN NUEVO POSGRADO DE NEGOCIOS CON UN ENFOQUE DE INNOVACIÓN, COMPETITIVIDAD, RESPONSABILIDAD SOCIAL Y ÉTICA EMPRESARIAL

\section{Objetivo}

Desarrollar un programa de Maestría que cumpla con los requerimientos de las instancias certificadoras y acreditadoras a nivel nacional e internacional, así como con el PLADE de la FCA UASLP y del PIDE de la FCA UASLP y que provea a los estudiantes de las competencias necesarias para dar solución a los problemas de los sectores productivos, para que las organizaciones participen con éxito en los mercados globalizados, con un enfoque de sustentabilidad, ética y responsabilidad social empresarial.

\section{Justificación}

Los posgrados enfocados al área de negocios y/o administración deben responder a los nuevos requerimientos y ofertar programas de formación pertinentes que integren a su currículo los conocimientos, habilidades transversales, valores y tendencias que rigen en el campo empresarial de tal modo que los egresados propongan soluciones que tomen en cuenta la ética y responsabilidad social que sean factibles en las organizaciones en las que se desenvuelven.

\section{Marco teórico}

\section{Tendencias a nivel nacional de los programas de posgrado de negocios}

Durante la Asamblea Nacional LV efectuada en junio de 2014, ANFECA mostró los resultados de un diagnóstico de los programas de posgrado de negocios llevado a cabo en las siete zonas geográficas del país. Habiendo aplicado un instrumento cuyo objetivo es conocer la visión de los diferentes grupos de interés de las IES afiliadas a la ANFECA (exalumnos, empleadores, empresarios y profesores) con relación a las tendencias demográficas, socioculturales, educativas, tecnológicas y económicas que visualizan para los siguientes 10 años, con la intención de identificar los conocimientos, las habilidades y las competencias requeridas en los futuros egresados. Este instrumento fue contestado por 211 personas, de las cuales 24 fueron empleadores del área de recursos humanos, 20 empleadores del área financiera, 25 empresarios, 57 exalumnos de posgrados de negocios de las diversas instituciones y 85 profesores. 
DISEÑO CURRICULAR DE UN NUEVO POSGRADO DE NEGOCIOS CON UN ENFOQUE DE INNOVACIÓN, COMPETITIVIDAD, RESPONSABILIDAD SOCIAL Y ÉTICA EMPRESARIAL

Los resultados de la aplicación de este instrumento evidenciaron las tendencias demográficas, socioculturales, económicas, tecnológicas y educativas que los posgrados de negocios deben observar en sus programas educativos.

\section{a. Tendencias demográficas}

Incluir temas relacionados con la forma de entender estas tendencias para enfrentar los retos que esto representa y al mismo tiempo detectar y capitalizar oportunidades de desarrollo económico.

- Se desarrolle una visión en los estudiantes sobre la sustentabilidad, que busque el equilibrio entre el desarrollo económico, la equidad social y el cuidado al medio ambiente.

- Se incluyan temas que permitan a los egresados realizar emprendimientos sociales que contribuyan al aprovechamiento de las oportunidades y enfrentar los retos derivados de estos cambios demográficos.

- Se impulse el emprendimiento para generar el desarrollo humano, como alternativa ante la falta de oportunidades profesionales que se vislumbra para los siguientes años.

b. Tendencias socioculturales

- Se fomente un pensamiento reflexivo, ético y ciudadano, con la intención de formar egresados socialmente responsables que contribuyan a solucionar estos problemas.

- Se fomente en los estudiantes el desarrollo de una mayor tolerancia y respeto a otras culturas, con la finalidad de comprender otras formas de pensar y de realizar negocios.

c. Tendencias tecnológicas

- Se haga una adecuación a la oferta de cursos y se ofrezcan algunos de ellos en formato virtual, con la intención de potenciar el uso de la tecnología y, al mismo tiempo, propiciar la flexibilidad y promover el trabajo asíncrono y a distancia. 
DISEÑO CURRICULAR DE UN NUEVO POSGRADO DE NEGOCIOS CON UN ENFOQUE DE INNOVACIÓN, COMPETITIVIDAD, RESPONSABILIDAD SOCIAL Y ÉTICA EMPRESARIAL

- Se incluyan temas relacionados con el análisis profundo de datos ("business analytics") con la intención de fomentar la toma de decisiones con base en el análisis detallado de información.

- Se incluyan contenidos relacionados con la forma de potenciar el uso de las TIC's y simuladores de negocios, para hacer más eficientes los procesos, facilitar los análisis y agilizar la toma de decisiones.

- Se incorporen con mayor intensidad temas relacionados con el emprendimiento para enfrentar los problemas de desempleo generados por los avances tecnológicos.

d. Tendencias educativas

- Se fomenten más habilidades de autoaprendizaje y que los momentos de interacción física en el aula se aprovechen para detonar el pensamiento crítico y el pensamiento creativo para proponer soluciones a los problemas sociales que enfrentan las comunidades (desigualdad social y económica, inseguridad, altas concentraciones urbanas en ciertas zonas, entre otros).

- Se haga más énfasis en el desarrollo de habilidades y competencias que permitan desarrollar capacidades visionarias con la finalidad de anticipar problemas y aprovechar oportunidades.

- Se fomente más la investigación y la realización de trabajos de campo que permitan solucionar problemas de interés para la comunidad empresarial y contribuyan a proponer soluciones a muchos de los problemas sociales que enfrentamos.

- Se explore la posibilidad de hacer alianzas estratégicas con universidades de Estados Unidos para ofrecer programas de posgrado conjuntos.

e. Tendencias económicas

- Se desarrollen habilidades relacionadas con el entendimiento y respeto de otras culturas, con la finalidad de adecuar las formas de negociar y detonar nuevos negocios.

- Se fomente la creatividad, la innovación y el emprendimiento para capitalizar las oportunidades derivadas de la formación de corredores industriales especializados en diversas zonas del país. 
DISEÑO CURRICULAR DE UN NUEVO POSGRADO DE NEGOCIOS CON UN ENFOQUE DE INNOVACIÓN, COMPETITIVIDAD, RESPONSABILIDAD SOCIAL Y ÉTICA EMPRESARIAL

- Se enfatice en temas relacionados con el desarrollo económico regional, con la intención de que los futuros egresados visualicen la relevancia de detonar proyectos que se inserten en las cadenas productivas actuales y futuras.

- Se incluyan debates y dilemas éticos en los negocios y en la práctica empresarial que preparen a los estudiantes para tomar decisiones apropiadas, basadas en la reflexión, en la cultura de la legalidad y que propicien el bienestar para la sociedad.

- Se profundice en el análisis y reflexión sobre nuevos modelos económicos que propicien la sustentabilidad social, económica y ambiental.

- Se incluyan formalmente temas relacionados con la ética, la ciudadanía y el desarrollo sustentable, que propicien en los estudiantes la reflexión que les permita identificar soluciones a muchos de los problemas que se viven en la actualidad, consecuencia del modelo económico vigente.

- Se potencie el liderazgo en los estudiantes, para que sean agentes de cambio en sus comunidades y en las organizaciones en las que colaboran (Zavaleta-Vázquez O., 2014).

\section{MATERIAL Y MÉTODO}

\section{Análisis nacional y regional de la oferta de posgrados de negocios con registro PNPC CONACYT}

Antes de proponer un programa nuevo de Maestría, se realizó un análisis comparativo de los programas ofertados a nivel regional y nacional de los posgrados en el área administrativa, que cuentan con el registro ante CONACYT dentro del PNPC, con el objetivo de identificar la estructura curricular de los programas de Maestría, su duración, número de materias, perfiles de ingreso y de egreso, así como sus objetivos.

\section{Análisis local de la oferta del posgrado de negocios}

También se llevó a cabo un estudio de la oferta de programas de posgrado en las áreas administrativas, contable, financiera y de gestión pública en la Ciudad de San Luis Potosí, con el fin de explorar un poco más las diferentes opciones de posgrado a nivel local, se realizó un estudio comparativo de las variables: costo total del programa, costo por materia, número de materias que incluye cada programa, duración y forma en que se imparte el programa. 
DISEÑO CURRICULAR DE UN NUEVO POSGRADO DE NEGOCIOS CON UN ENFOQUE DE INNOVACIÓN, COMPETITIVIDAD, RESPONSABILIDAD SOCIAL Y ÉTICA EMPRESARIAL

\section{Análisis local de la demanda de posgrados de negocios}

Como parte de la evaluación de la demanda de los diferentes posgrados de negocios, se incluyó el estudio (Castañón Nieto E., Azuara Pugliese, V. y Ávila Ortega, M. del C., 2013) con egresados de la FCA-UASLP el cual se realizó en el 2012 con una muestra de 193 estudiantes de último semestre de las profesiones de Contaduría Pública, Licenciados en Administración y Licenciados en Administración Pública de la FCA-UASLP, utilizando el modelo BrisouxLaroche, en el cual se les preguntó no solo sus intenciones de estudiar un posgrado, sino las características decisivas para decidir por un programa de posgrado específico.

\section{Consulta a expertos, empleadores y profesores de la FCA-UASLP}

En cuanto a las consultas a expertos, empleadores y profesores de la FCA, se realizaron tres foros: uno en diciembre 2012, otro en febrero 2013, y el último en abril 2013, a los que fueron invitados profesionistas, académicos y cámaras de comercio, así como alumnos y egresados, para que externaran su opinión y retroalimentación en relación a la estructura curricular y pertinencia en la apertura del nuevo programa.

\section{Análisis del perfil de ingreso de las maestrías que ofrece la FCA UASLP}

Se realizó también un análisis del perfil de ingreso de los estudiantes a la Maestría en Administración con énfasis en Negocios que se ofrece actualmente, durante el periodo 20082014.

\section{RESULTADOS}

\section{Oferta Nacional y Regional de posgrados PNPC CONACYT}

Como resultado del análisis realizado de la oferta nacional y regional de posgrados con registro PNPC CONACYT (ver tabla 1), el número de cursos de los programas oscilan entre 12 y 24 materias, en cuanto a su duración, expresada en trimestres, cuatrimestres o semestres según sea el caso, la mayoría tiene un plazo entre dos y dos años y medio, todas son profesionalizantes, lo que hace ver claramente la tendencia de enfocarse hacia el mercado de los negocios, no así hacia la investigación. Por otro lado, se observa que existen pocos programas de Maestría con registro PNPC, ubicados en la zona centro del país, justificando 
DISEÑO CURRICULAR DE UN NUEVO POSGRADO DE NEGOCIOS CON UN ENFOQUE DE INNOVACIÓN, COMPETITIVIDAD, RESPONSABILIDAD SOCIAL Y ÉTICA EMPRESARIAL

con esto la creación de este nuevo programa en el PNPC en la UASLP en el área de la administración en la región centro.

Asimismo, se presenta en la tabla 2, la oferta de Maestría que ofrecen programas similares registrados en el PNPC a nivel nacional, pudiendo observar junto con las Maestrías en Políticas Públicas y afines, la Maestría en Administración y/o Negocios, son por mucho, la mayoría del total de posgrados.

Para complementar el análisis de pertinencia, se tomó como referencia el estudio realizado por Zavaleta-Vázquez (2014) quien realiza un comparativo del estado que guardan los posgrados de las instituciones afiliadas a la Asociación Nacional de Facultades y Escuelas de Contaduría y Administración (ANFECA) y su demanda a nivel nacional (ver tabla 3), donde se observa que la Maestría en Administración sigue siendo la más demandada para estudiar un posgrado, en donde se muestra el promedio de alumnos por institución; $\mathrm{N}$ representa el número de instituciones bajo estudio.

\section{Análisis local de la oferta del posgrado de negocios}

En el estudio de las ofertas de programas de posgrado en las áreas administrativas, contable, financiera y de gestión pública, en la Ciudad de San Luis Potosí, se obtuvieron los datos que se observan en la tabla 4. De los 31 programas de posgrado, nueve son los que están enfocados al área de la administración, en donde participan la Universidad del Valle de México, con dos programas, una Maestría en Administración y un MBA, la Universidad de Estudios Avanzados, con un programa de Maestría en Administración, la Universidad Tangamanga, con dos Maestrías en las Áreas de Negocios y Administración, el Instituto Tecnológico y de Estudios Superiores de Monterrey, quien ofrece los programas de Maestrías similares en línea; es decir, no presenciales, la Universidad Interamericana para el Desarrollo, con su Maestría en Administración de Negocios, además de la UASLP con la Maestría en Administración con énfasis en Negocios. Cabe mencionar, que estos posgrados en el área de negocios representan casi el 30\% del total de posgrados ofertados por diferentes Universidades en la Ciudad de San Luis Potosí.

El resultado del estudio comparativo de las variables: costo total del programa, costo por materia, número de materias que incluye cada programa, duración y forma en que se imparte el programa (ver tabla 5); el programa de Maestría que ofrece la PFAC-UASLP, sigue siendo el menos costoso aunque el costo por materia es un poco más alto que el de la Maestría en 
DISEÑO CURRICULAR DE UN NUEVO POSGRADO DE NEGOCIOS CON UN ENFOQUE DE INNOVACIÓN, COMPETITIVIDAD, RESPONSABILIDAD SOCIAL Y ÉTICA EMPRESARIAL

Planeación Estratégica e Innovación ofrecido por la Facultad de Ingeniería UASLP, ésta cuenta con 19 materias, mientras que la de Administración tiene 14.

En cuanto a la duración del programa, la UNID, el Tec Milenio y la UNEA la ofrecen en un año ocho meses, la UVM, la Facultad de Ingeniería UASLP y la UTAN en dos años, el ITESM en dos años y medio, mientras que la FCA UASLP en dos años y un cuatrimestre.

Cabe mencionar, que de estos programas de maestría relacionados con el área administrativa que se ofrecen en la ciudad de San Luis Potosí, ninguno de ellos pertenece al PNPC.

\section{Análisis local de la demanda del posgrado de negocios}

Los resultados del estudio (Castañón Nieto E., Azuara Pugliese, V. y Ávila Ortega, M. del C., 2013) con egresados de la FCA-UASLP con una muestra de 193 egresados en el 2012 de las profesiones de Contaduría Pública, Licenciados en Administración y Licenciados en Administración Pública de la FAC-UASLP. El instrumento incluyó una pregunta sobre sus deseos de estudiar una Maestría, 84 personas contestaron SI (44\%) y 109 personas NO (56\%). De los 84 que desean estudiar una Maestría 58 (69\%) son mujeres y 27 (32\%) son hombres.

Al pedirles que mencionaran las instituciones que supieran que ofrecen estudios de posgrado (maestrías específicamente) en el área socio-económica-administrativa, en la ciudad de San Luis Potosí, sin ofrecer una lista de opciones, en promedio recordaron 2.94 IES y la frecuencia de mención se ve reflejada en la figura 1, donde destaca con 59 menciones, la UASLP, seguida del Instituto Tecnológico de Estudios Superiores de Monterrey, con 45 y la Universidad del Valle con 43.

El egresado de la licenciatura o aquellos interesados en obtener un grado de maestro, tienen una gama de alternativas para elegir su programa de estudio. Las siguientes instituciones ofertan programas de posgrado en la ciudad de San Luis Potosí:

1. Universidad Autónoma de San Luis Potosí (UASLP).

2. Colegio de San Luis A.C. (COLSAN).

3. Instituto Tecnológico y de Estudios Superiores de Monterrey (ITESM).

4. Universidad Cuauhtémoc.

5. Universidad Marista (Champagnat).

6. Universidad del Centro de México (UCEM).

7. Universidad del Valle de México (UVM). 
DISEÑO CURRICULAR DE UN NUEVO POSGRADO DE NEGOCIOS CON UN ENFOQUE DE INNOVACIÓN, COMPETITIVIDAD, RESPONSABILIDAD SOCIAL Y ÉTICA EMPRESARIAL

8. Universidad de Estudios Avanzados (UNEA), antes Interamericana del Norte.

9. Universidad Interamericana para el Desarrollo (UNID).

10. Universidad Tangamanga (UTAN).

11. Universidad Tec Milenio.

Una vez identificados los factores de importancia al momento de seleccionar un programa de posgrado, se les indicó elegir entre los programas ofertados en las diferentes IES. La Maestría en Administración con énfasis en Negocios ofertada por la UASLP sigue siendo la primera opción de los egresados de licenciatura, frente al resto de las IES que ofrecen programas similares (ver figura 2).

A fin de justificar el nuevo programa de Maestría se realizó un estudio que identifica los atributos decisivos para elegir un programa de posgrado. De acuerdo con Castañón Nieto E., Azuara Pugliese, V. y Ávila Ortega, M. del C. (2013), los estudiantes de licenciatura próximos a egresar en el área socio-económico-administrativa, confirman que los factores decisivos para elegir un programa de posgrado son, en primer lugar, el tipo de oferta educativa; y en segundo lugar, el nivel académico percibido. Asimismo, consideran que el precio y las opciones de obtención del grado académico son factores de alta relevancia en su decisión. En este sentido, de una muestra de 193 estudiantes del último semestre de las carreras de Contaduría Pública, Licenciados en Administración y Licenciados en Administración Pública, el 30.5\% tiene preferencia por los programas ofrecidos por la UASLP, el 23.3\% por los programas ofrecidos en el ITESM, el $22.2 \%$ se inclinan por los posgrados de la UVM y el $10.8 \%$ por la Universidad Tangamanga. Del total de encuestados 84 personas (44\%), están interesadas en continuar sus estudios de posgrado, de los cuales el 74\% elegirían como primera opción a la UASLP, seguida del ITESM con un 4\%. Ello, sugiere el mercado potencial que hay en la propia Facultad de Contaduría y Administración de la UASLP.

Los factores, en orden de importancia, que los interesados en estudiar un programa de posgrado consideran más importantes al seleccionar un programa son los siguientes:

1. Ofrece la maestría que quiero estudiar (32/84).

2. Prestigio de la Institución (26/84).

3. Preparación académica del profesorado (21/84).

4. Calidad reconocida por los empleadores (17/84). 
DISEÑO CURRICULAR DE UN NUEVO POSGRADO DE NEGOCIOS CON UN ENFOQUE DE INNOVACIÓN, COMPETITIVIDAD, RESPONSABILIDAD SOCIAL Y ÉTICA EMPRESARIAL

5. Precio acorde a mis posibilidades (13/84).

6. Que esté certificada por el PNPC, CONACYT (13/84).

7. Plan curricular (12/84).

8. Con opciones para obtener el grado (9/84).

9. Horarios flexibles para trabajadores (9/84).

10. Programas de intercambio y movilidad (6/84).

11. Tiempo corto de estudio (6/84).

12. Cursos de fin de semana (6/84).

13. Instalaciones (4/84).

14. Tamaño de la institución (4/84).

15. Ubicación geográfica (4/84).

16. Ofrezcan cursos en línea (3/84).

Se observó, que uno de los principales atributos de la oferta de Maestrías en la que los egresados de licenciatura de la FCA-UASLP basan su decisión de elección de IES, es precisamente que cuente con la Maestría que desean estudiar, el prestigio de la IES, la preparación académica del profesorado, de la calidad reconocida por parte de los empleadores y del precio acorde a sus posibilidades, seguida de que cuente con la certificación del PNPC de CONACYT.

Si bien, la gran fortaleza de los posgrados de la FCA, UASLP es el prestigio de la Institución, la cuidada selección del profesorado, el reconocimiento por parte de los empleadores y el precio, es necesario que contrarreste algunas de sus debilidades, implementando estrategias que se enuncian como fortalezas en otras instituciones y no se poseen en ésta, tal es el caso de la certificación en el PNPC, clases en fines de semana, programas de intercambio y movilidad estudiantil y alguna materia en línea; lo que se consideraba como una gran desventaja al exigir tesis y examen de grado, solo obtuvo 9 menciones.

\section{Resultados de la consulta a expertos, empleadores y profesores de la FCA UASLP}

El resultado de los tres foros a los que fueron invitados profesionistas, académicos y cámaras de comercio, así como alumnos y egresados, para que externaran su opinión y retroalimentación en relación a la estructura curricular y pertinencia en la apertura del nuevo programa. En este sentido, se llegó a un consenso en cuanto al nombre del programa, siendo 
DISEÑO CURRICULAR DE UN NUEVO POSGRADO DE NEGOCIOS CON UN ENFOQUE DE INNOVACIÓN, COMPETITIVIDAD, RESPONSABILIDAD SOCIAL Y ÉTICA EMPRESARIAL

más atractivo e integral el nombre de Maestría en Dirección Empresarial. Asimismo, las diferentes personas participaron en la validación del plan curricular, la pertinencia de la inclusión de cursos y seminarios, así como las áreas de concentración del programa, el número de materias, los requisitos académicos y los perfiles de ingreso y egreso.

\section{Análisis del perfil de ingreso de las Maestrías que ofrece la FCA UASLP}

El resultado del análisis del perfil de ingreso de los estudiantes a la Maestría en Administración con énfasis en Negocios que se ofrece actualmente, durante el periodo 2008-2014 (ver figura 4), el 35.5\% son ingenieros, $17.8 \%$ licenciados en administración, 15.9\% contadores públicos, 8.4\% licenciados en comercio exterior y el 22.4\% provienen de 11 carreras diferentes. Esto pone en evidencia la multidisciplinariedad de los alumnos, pero también la gran demanda que tiene la Maestría por parte de los ingenieros que se encuentran insertados en el sector industrial.

Los registros de la División de Estudios de Posgrado de la FCA-UASLP muestran que cerca del $44 \%$ de los estudiantes de la Maestría en Administración con énfasis en Negocios se encuentran trabajando en el sector industrial, el $14.95 \%$ en Instituciones de Educación Superior, el $11.2 \%$ en el sector de servicios, $7.48 \%$ en el sector comercial, un $8.4 \%$ en otros sectores y el $11.2 \%$ se dedica solo a estudiar (ver figura 5 ). Por lo tanto, casi el $90 \%$ de los estudiantes se encuentran insertados en el mercado laboral y de ellos el 50\% prestan sus servicios en el sector industrial, lo cual justifica plenamente la propuesta de que el nuevo programa de Maestría en Dirección Empresarial se registre ante CONACYT dentro del PNPC en su modalidad profesionalizante.

\section{CONCLUSIONES}

Al tenor de lo expuesto, la División de Estudios de Posgrado de la FCA, consideró pertinente ofrecer un nuevo programa de Maestría en Dirección Empresarial, particularmente como un programa de posgrado profesionalizante, aprovechando esta oportunidad en el mercado, además de que San Luis Potosí, por su ubicación geográfica y crecimiento industrial, comercial y de servicios en los últimos años, requiere de posgraduados de programas con estas características y atendiendo los lineamientos del modelo CONACYT de referencia. 
DISEÑO CURRICULAR DE UN NUEVO POSGRADO DE NEGOCIOS CON UN ENFOQUE DE INNOVACIÓN, COMPETITIVIDAD, RESPONSABILIDAD SOCIAL Y ÉTICA EMPRESARIAL

Una vez realizado el estudio comparativo de la oferta local y nacional en programas de Maestrías en el área administrativa inscritos en el PNPC y la investigación de campo con los egresados de las diferentes carreras de la FCA-UASLP que formó parte de la evaluación para la inclusión de la nueva oferta educativa, y con una idea clara del posicionamiento que tiene el posgrado en la mente de los egresados, se procedió a evaluar la inclusión de la Maestría en Dirección Empresarial en la nueva oferta de posgrado de la División de Estudios de Posgrado de la FCA-UASLP.

Además atenderá la flexibilidad requerida por el mercado laboral actual, al integrar las áreas de concentración de estrategia empresarial y competitividad y desarrollo del capital humano, en donde los estudiantes podrán seleccionar dos cursos electivos de acuerdo a su área de especialización.

Consecuentemente, el programa de Maestría en Dirección Empresarial proporcionará al estudiante los conocimientos y habilidades para la Dirección Empresarial con un enfoque de competitividad, ética y responsabilidad social de acuerdo a la actualidad multicultural del mundo empresarial como se muestra en la figura 5.

Tabla 1

Maestrías en áreas administrativas inscritas en el Programa Nacional de Posgrados de Calidad (PNPC) a nivel regional y nacional

\begin{tabular}{|c|c|c|c|c|c|}
\hline Programa & Institución & Entidad & Orientación & $\begin{array}{l}\text { Número } \\
\text { Materias }\end{array}$ & Duración \\
\hline Maestría en Administración & $\begin{array}{c}\text { Centro de Enseñanza Técnica } \\
\text { y Superior }\end{array}$ & Baja California & Profesionalizante & 14 & 2 Años \\
\hline Maestría en Administración & $\begin{array}{l}\text { Instituto Politécnico } \\
\text { Nacional }\end{array}$ & Distrito Federal & Profesionalizante & 12 & 2 Años \\
\hline Maestría en Administración & $\begin{array}{l}\text { Instituto Tecnológico y de } \\
\text { Estudios Superiores } \\
\text { de Monterrey }\end{array}$ & Chihuahua & Profesionalizante & 18 & 2 Años \\
\hline $\begin{array}{l}\text { Maestría en Administración } \\
\text { y Dirección de Empresas } \\
\text { (Monterrey) }\end{array}$ & $\begin{array}{l}\text { Instituto Tecnológico y de } \\
\text { Estudios Superiores de } \\
\text { Monterrey }\end{array}$ & Nuevo León & Profesionalizante & 17 & - \\
\hline Maestría en Administración & $\begin{array}{l}\text { Universidad Autónoma de } \\
\text { Ciudad Juárez }\end{array}$ & Chihuahua & Profesionalizante & 15 & 2 Años \\
\hline Maestría en Administración & $\begin{array}{l}\text { Universidad Autónoma del } \\
\text { Estado de Hidalgo }\end{array}$ & Hidalgo & Profesionalizante & 19 & 2.5 Años \\
\hline
\end{tabular}


DISEÑO CURRICULAR DE UN NUEVO POSGRADO DE NEGOCIOS CON UN ENFOQUE DE INNOVACIÓN, COMPETITIVIDAD, RESPONSABILIDAD SOCIAL Y ÉTICA EMPRESARIAL

\begin{tabular}{|c|c|c|c|c|c|}
\hline $\begin{array}{l}\text { Maestría en Administración } \\
\text { de Negocios }\end{array}$ & Universidad de Guadalajara & Jalisco & Profesionalizante & 13 & 2 Años \\
\hline $\begin{array}{l}\text { Maestría en } \\
\text { Administración }\end{array}$ & $\begin{array}{l}\text { Universidad de } \\
\text { Guanajuato }\end{array}$ & Guanajuato & Profesionalizante & 16 & 2 Años \\
\hline $\begin{array}{l}\text { Maestría en Ciencias } \\
\text { Económico y } \\
\text { Administrativas }\end{array}$ & $\begin{array}{c}\text { Universidad Autónoma de } \\
\text { Aguascalientes }\end{array}$ & Aguascalientes & Profesionalizante & 20 & 2 Años \\
\hline $\begin{array}{l}\text { Programa Master en } \\
\text { Dirección de Empresas }\end{array}$ & $\begin{array}{c}\text { Sociedad Panamericana de } \\
\text { Estudios } \\
\text { Empresariales A.C. }\end{array}$ & Distrito Federal & Profesionalizante & - & $\begin{array}{l}1 \text { Año } 10 \\
\text { Meses }\end{array}$ \\
\hline Maestría en Administración & $\begin{array}{c}\text { Universidad Autónoma de } \\
\text { Baja } \\
\text { California }\end{array}$ & Baja California & Profesionalizante & - & 2 Años \\
\hline $\begin{array}{l}\text { Maestría en Administración } \\
\text { y Alta Dirección }\end{array}$ & $\begin{array}{l}\text { Universidad Autónoma de } \\
\text { Coahuila }\end{array}$ & Coahuila & Profesionalizante & 24 & 2 Años \\
\hline $\begin{array}{l}\text { Maestría en Administración } \\
\text { Estratégica }\end{array}$ & $\begin{array}{l}\text { Universidad Autónoma de } \\
\text { Sinaloa }\end{array}$ & Sinaloa & Profesionalizante & 16 & 2 Años \\
\hline $\begin{array}{l}\text { Maestría en Dirección } \\
\text { Empresarial }\end{array}$ & $\begin{array}{c}\text { Universidad Autónoma de } \\
\text { Tamaulipas }\end{array}$ & Tamaulipas & Profesionalizante & 15 & $\begin{array}{l}1 \text { Año } 8 \\
\text { Meses }\end{array}$ \\
\hline Maestría en Administración & $\begin{array}{l}\text { Universidad Autónoma del } \\
\text { Estado de México }\end{array}$ & $\begin{array}{l}\text { Estado de } \\
\text { México }\end{array}$ & Profesionalizante & 15 & 2 Años \\
\hline $\begin{array}{l}\text { Maestría en Gestión y } \\
\text { Desarrollo Empresarial }\end{array}$ & Universidad de Occidente & Sinaloa & Profesionalizante & 17 & 2 Años \\
\hline
\end{tabular}

Fuente: CONACYT (Abril, 2015). Programa Nacional de Posgados de Calidad (PNPC).

Marco de referencia para la evaluación y seguimiento de programas de posgrado presenciales. México: CONACYT. Subsecretaría de Educación Superior.

Tabla 2

Oferta de posgrados similares a la oferta del Posgrado de la FCA-UASLP a nivel nacional inscritas en el PNPC

\begin{tabular}{lc}
\hline Programa PNPC 2015 & Total \\
\hline Maestrías en administración y/o negocios & 16 \\
Maestrías en mercadotecnia & 2 \\
Maestrías en recursos humanos & 1 \\
Maestrías en finanzas & 2 \\
Maestrías en negocios internacionales & 3 \\
Maestrías en política pública y afines & 11 \\
\hline
\end{tabular}

Fuente: Elaboración propia con datos de CONACYT (Abril, 2015).

Programa Nacional de Posgados de Calidad (PNPC). Marco de referencia para la evaluación y seguimiento de programas de posgrado presenciales. México: CONACYT. Subsecretaría de Educación Superior. 
DISEÑO CURRICULAR DE UN NUEVO POSGRADO DE NEGOCIOS CON UN ENFOQUE DE INNOVACIÓN, COMPETITIVIDAD, RESPONSABILIDAD SOCIAL Y ÉTICA EMPRESARIAL

Tabla 3

Demanda de programas de posgrados a nivel nacional en IES afiliadas a ANFECA

\begin{tabular}{|l|c|c|c|c|}
\hline \multicolumn{5}{|c|}{ Alumnos inscritos } \\
\hline & Media & Mínimo & Máximo & $\mathrm{N}$ \\
\hline Maestría en Administración & 172 & 0 & 1709 & 40 \\
\hline Maestría en Finanzas & $\overline{88}$ & 0 & 925 & 30 \\
\hline Maestría en Mercadotecnia & 20 & 0 & 121 & 23 \\
\hline Maestría en Negocios Internacionales & 55 & 0 & 411 & 17 \\
\hline Maestría en Contaduría & 161 & 0 & 1719 & 12 \\
\hline Doctorado en Administración & 34 & 0 & 215 & 23 \\
\hline Otro & 98 & 0 & 967 & 39 \\
\hline
\end{tabular}

Fuente: Zavaleta-Vázquez O. H. (junio, 2014). El Posgrado en las Instituciones de Educación Superior Afiliadas a la ANFECA.

Conferencia Magistral durante la LV Asamblea Nacional de ANFECA León Guanajuato. Recuperada de http://www.anfeca.unam.mx/docs/tema_central/tema_central.pdf

Tabla 4

Oferta de Maestrías del área contable-administrativa en la ciudad de San Luis Potosí

1. Maestría en Administración. UVM

2. Maestría en Administración. UNEA

3. Maestría en Administración. UTAN

4. Maestría en Administración Empresarial (en línea). ITESM

5. Maestría en Administración de Negocios. UVM

6. Maestría en Administración de Negocios. UNID

7. Maestría en Administración de Negocios. UTAN

8. Maestría en Administración con énfasis en Negocios. U.A.S.L.P.

9. Maestría en Administración de Negocios Globales (en línea). ITESM

10. Maestría en Finanzas (en línea). ITESM

11. Maestría en Finanzas. U. MARISTA

12. Maestría en Administración de Negocios en Finanzas. U. TECMILENIO

13. Maestría en Mercadotecnia (en línea). ITESM

14. Maestría en Mercadotecnia. UNID

15. Maestría en Administración de Negocios en Mercadotecnia. U. TECMILENIO

16. Maestría en Mercadotecnia de Negocios Turísticos. UVM
17. Maestría en Administración de Negocios en Calidad y Productividad. U. TecMILENIO

18. Maestría en Administración Industrial. UTAN

19. Maestría en Planeación Estratégica e Innovación. U.A.S.L.P.

20. Maestría en Administración de Negocios en Recursos Humanos. U. TecMILENIO

21. Maestría en Desarrollo Humano. U. MARISTA

22. Maestría en Alta Dirección. U.A.S.L.P.-U.QUEBEC

23. Maestría en Comercio Electrónico (en línea). ITESM

24. Maestría en Comercio Exterior. UVM

25. Maestría en Comercio Internacional. UCEM

26. Maestría en Administración con énfasis en Impuestos. U.A.S.L.P.

27. Maestría en Derecho Fiscal y Privado. U. MARISTA

28. Maestría en Derecho Fiscal. UVM

29. Maestría en Administración con énfasis en Gestión Pública. U.A.S.L.P.

30. Maestría en Asuntos Políticos y Políticas Públicas. COLSAN

31. Maestría en Gestión Pública Aplicada (en línea). ITESM

Fuente: Castañón Nieto E., Azuara Pugliese, V. y Ávila Ortega, M. del C. (2013). Análisis de los atributos de decisión en la elección de programas de posgrado del área socio ecnómico-administrativa en San Luis Potosí, por los egresados de la FCA-UASLP. $8^{\circ}$. Foro Nacional y 3er. Foro Internacional de la academia ANFECA, 1-30. 
DISEÑO CURRICULAR DE UN NUEVO POSGRADO DE NEGOCIOS CON UN ENFOQUE DE INNOVACIÓN, COMPETITIVIDAD, RESPONSABILIDAD SOCIAL Y ÉTICA EMPRESARIAL

Tabla 5

Comparativa de las maestrías locales (competencia directa)

\begin{tabular}{|c|c|c|c|c|c|}
\hline Programa & $\begin{array}{l}\text { Costo total } \\
\text { programa }\end{array}$ & Costo por materia & $\begin{array}{l}\text { Número de } \\
\text { materias }\end{array}$ & Duración programa & Duración de curso \\
\hline $\begin{array}{l}\text { TEC DE MONTERREY Maestria } \\
\text { en administración empresarial } \\
\text { (en linea) }\end{array}$ & $\$ 300,220$ & $\$ 17,660$ & 17 & 2 años 6 meses & Trimestral \\
\hline $\begin{array}{l}\text { UVM Maestria en administración } \\
\text { en negocios }\end{array}$ & $\$ 110,000$ & $\$ 6,000$ & 18 & 2 años & Cuatrimestral \\
\hline $\begin{array}{l}\text { UNID Maestria en } \\
\text { administración de negocios }\end{array}$ & $\$ 74,000$ & $\$ 5,333$ & 14 & 1 año 8 meses & Cuatrimestral \\
\hline $\begin{array}{l}\text { TEC MILENIO Maestria en } \\
\text { administración de negocios }\end{array}$ & $\$ 70,000$ & $\$ 4,666$ & 15 & 1 año 8 meses & Cuatrimestral \\
\hline $\begin{array}{l}\text { UTAN Maestria en } \\
\text { administración }\end{array}$ & $\$ 69,660$ & $\$ 3,666$ & 19 & 2 años & Cuatrimestral \\
\hline $\begin{array}{l}\text { UNEA Maestria en } \\
\text { administración }\end{array}$ & $\$ 66,600$ & $\$ 4,440$ & 15 & 1 año 8 meses & Cuatrimestral \\
\hline $\begin{array}{l}\text { UASLP Maestria en planeación } \\
\text { estratégica e innovación }\end{array}$ & $\$ 57,000$ & $\$ 3,000$ & 19 & TC 2 años y TP 3 años & Semestral \\
\hline $\begin{array}{l}\text { UASLP Maestria en } \\
\text { administración con énfasis en } \\
\text { negocios }\end{array}$ & $\$ 47,600$ & $\$ 3,400$ & 14 & 2 años y 4 meses & Cuatrimestral \\
\hline
\end{tabular}

Fuente: Elaboración propia con datos de 2012.

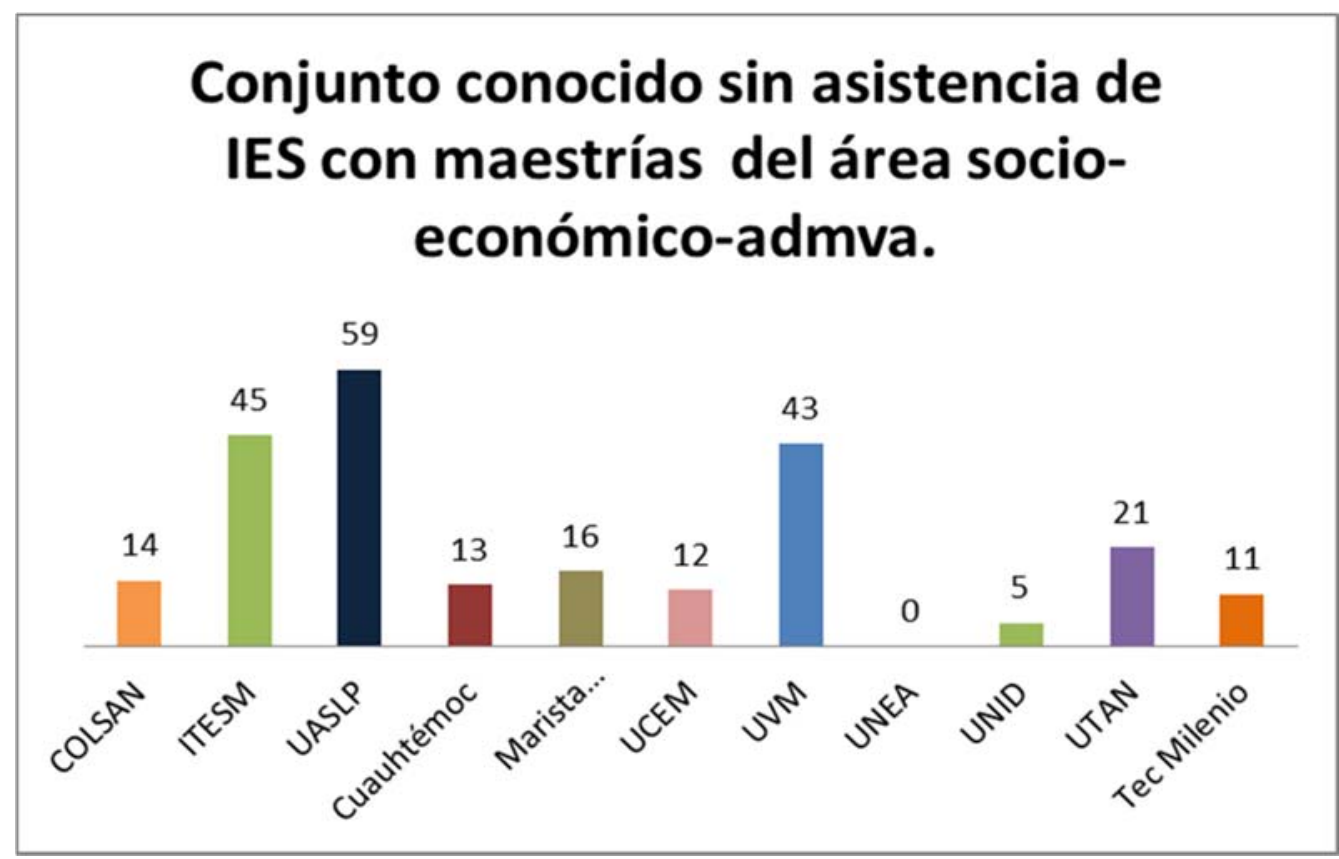

Figura 1. Conjunto conocido sin asistencia de IES con maestrías en el área socioadministrativa.

Fuente: Castañón Nieto, E., Azuara Pugliese, V. y Ávila Ortega M. del C. (2013). Análisis de los atributos de decisión en la elección de programas de posgrado del área socio ecnómico administrativa en San Luis Potosí, por los egresados de la FCA-UASLP. $8^{\circ}$. Foro Nacional y 3er. Foro Internacional de la academia ANFECA, 1-30. 
DISEÑO CURRICULAR DE UN NUEVO POSGRADO DE NEGOCIOS CON UN ENFOQUE DE INNOVACIÓN, COMPETITIVIDAD, RESPONSABILIDAD SOCIAL Y ÉTICA EMPRESARIAL

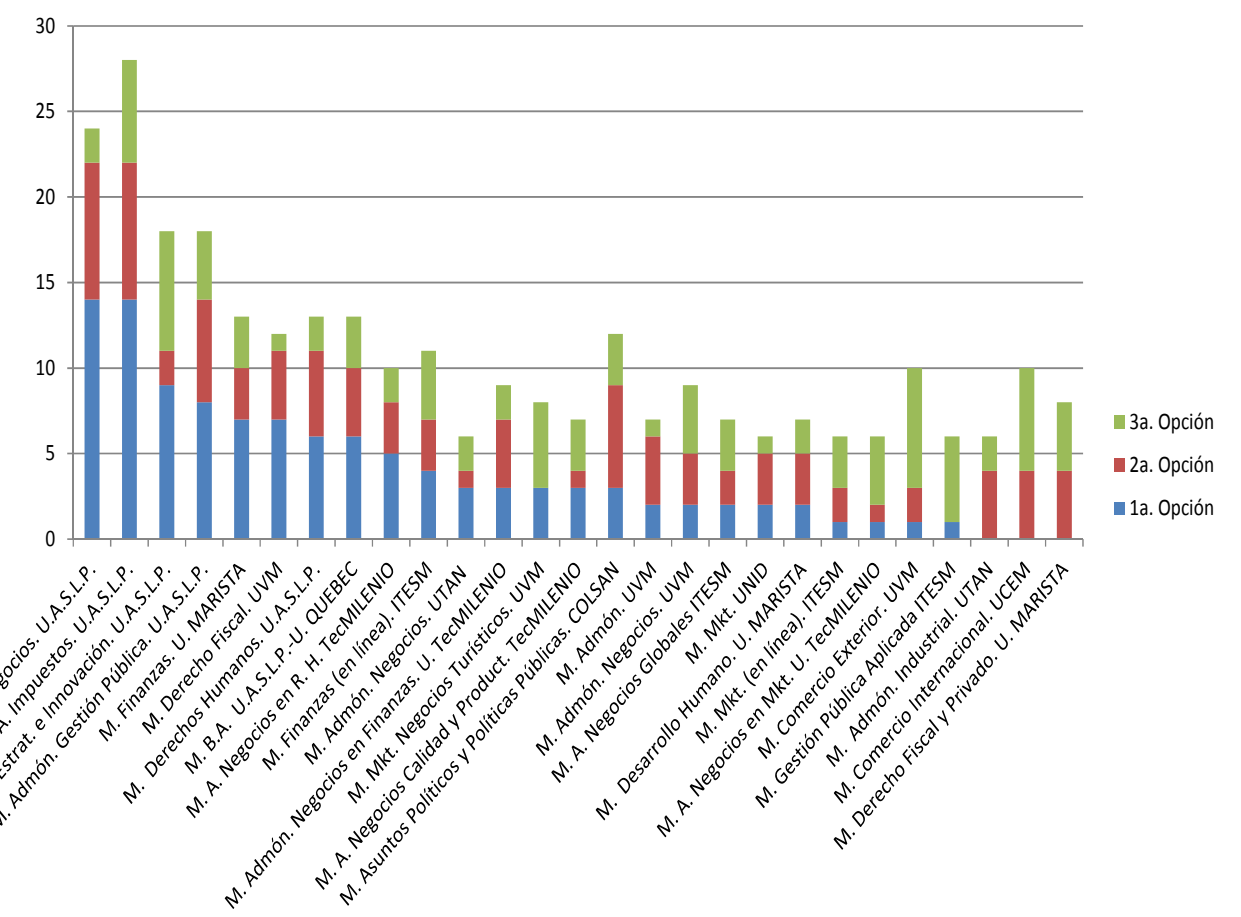

Figura 2. Maestrías ofertadas por las IES en San Luis Potosí1.

Fuente: Castañón Nieto, E., Azuara Pugliese, V. y Ávila Ortega, M. del C. (2013). Análisis de los atributos de decisión en la elección de programas de posgrado del área socio ecnómico-administrativa en San Luis Potosí, por los egresados de la FCA-UASLP. 8. Foro Nacional y 3er. Foro Internacional de la academia ANFECA, 1-30.

PERFIL DE ALUMNOS M.A.N. ENERO-ABRIL 2013
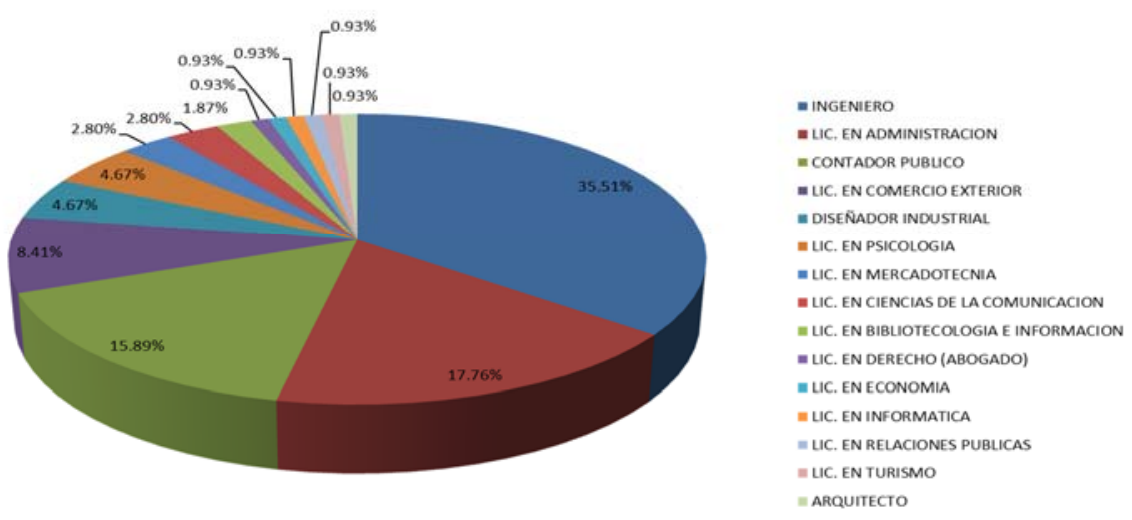

Figura 3. Perfil de los alumnos de la Maestría en Administración con énfasis en Negocios enero-abril 2013.

Fuente: Elaboración basada en registros de control escolar de la División de Estudios de Posgrado de la FCA-UASLP.

${ }^{1}$ (De las 34 opciones de maestrías se eliminaron en el gráfico aquellas que tuvieron menos de 5 menciones en total). 
DISEÑO CURRICULAR DE UN NUEVO POSGRADO DE NEGOCIOS CON UN ENFOQUE DE INNOVACIÓN, COMPETITIVIDAD, RESPONSABILIDAD SOCIAL Y ÉTICA EMPRESARIAL

SECTOR LABORAL

ALUMNOS M.A.N. ENERO-ABRIL 2013

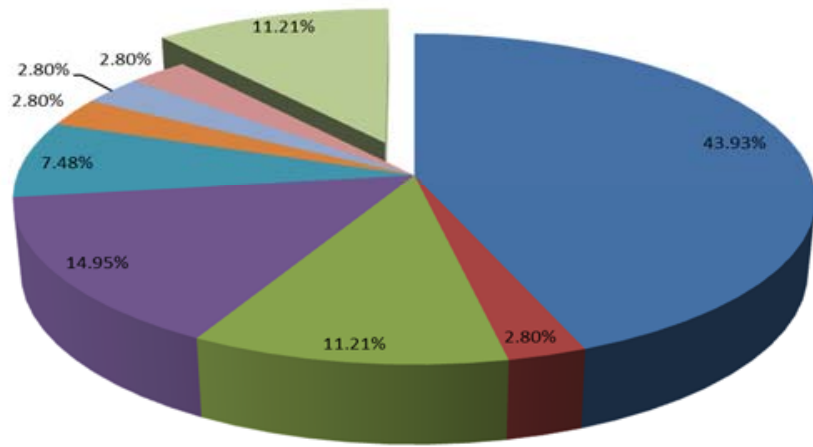

$=$ INDUSTRIA

= BANCA

= SERVICIOS

$=$ I.E.S.

- COMERCIO

= CONSTRUCCIÓN

= SECTOR PÚBLICO

= MICROEMPRESA

= NO TRABAIA

Figura 4. Sector laboral de los alumnos de la Maestría en Administración con énfasis en Negocios enero-abril 2013.

Fuente: Elaboración propia basada en registros de control escolar de la División de Estudios de Posgrado FCA-UASLP.

NIVEL DE CONCENTRACIÓN (2 CURSOS ELECTIVOS)

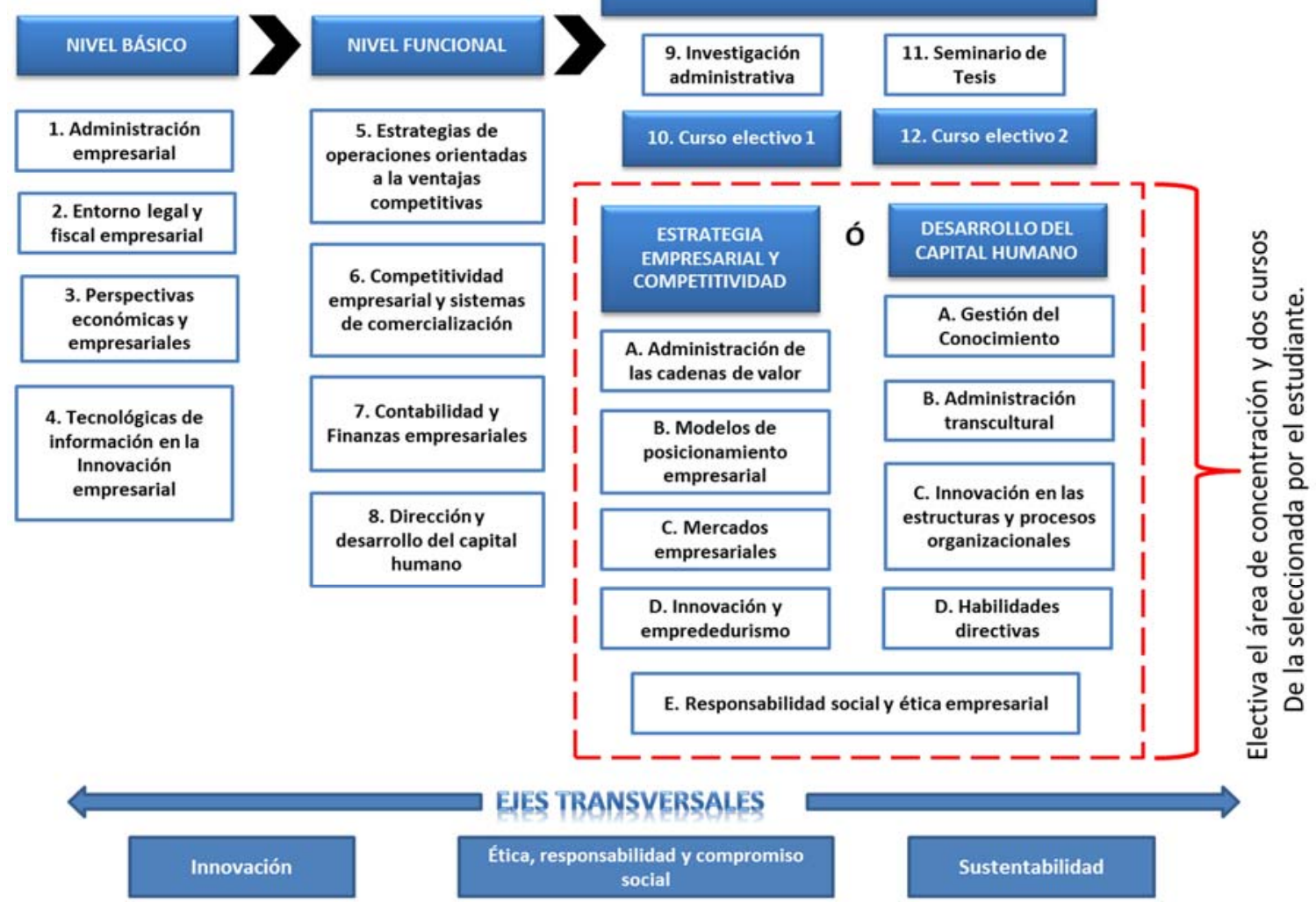

Figura 5. Mapa curricular de la Maestría en Dirección Empresarial.

Fuente: Elaboración propia. 
DISEÑO CURRICULAR DE UN NUEVO POSGRADO DE NEGOCIOS CON UN ENFOQUE DE INNOVACIÓN, COMPETITIVIDAD, RESPONSABILIDAD SOCIAL Y ÉTICA EMPRESARIAL

\section{REFERENCIAS BIBLIOGRÁFICAS}

Castañón Nieto, E., Azuara Pugliese, V., \& Ávila Ortega, M. del C. (marzo, 2013). Análisis de los atributos de decisión en la elección de programas de posgrado del área socio ecnómico-administrativa en San Luis Potosí, por los egresados de la FCA-UASLP. San Luis Potosí, S. L. P., 8. Foro Nacional y 3er. Foro Internacional de la academia ANFECA, El capital iintelectual como estrategia de la emprenduria en negocio, 1-30, ISBN 978-607-7856-89-4

CONACYT (Abril, 2015). Programa Nacional de Posgrados de Calidad (PNPC). Marco de referencia para la evaluación y seguimiento de programas de posgrado presenciales. México: CONACYT. Subsecretaría de Educación Superior.

Zavaleta-Vázquez O. H. (junio, 2014). El Posgrado en las Instituciones de Educación Superior Afiliadas a la ANFECA. Conferencia Magistral durante la LV Asamblea Nacional de ANFECA León

Guanajuato.

Recuperado

de http://www.anfeca.unam.mx/docs/tema_central/tema_central.pdf 